\title{
APUNTES ETNOZOOLÓGICOS: MONTES DE MARÍA, SUCRE, COLOMBIA
}

\section{ETHNOZOOLOGICAL NOTES: MONTES DE MARÍA, SUCRE, COLOMBIA}

\author{
ALEJANDRO DE LA OSSA-LACAYO,${ }^{1 *}$ M.Sc, JAIME DE LA OSSA V, ${ }^{2}$ Dr.
}

${ }^{1}$ CARSUCRE, Colombia.

${ }^{2}$ Universidad de Sucre, Grupo de Investigación en Biodiversidad Tropical, Colombia.

\section{Key words:}

Ethnozoology, wildlife,

Sucre,

Colombia.

\section{Abstract}

The present ethnozoological study evaluates the use given to the wildlife species that are common consumed by a rural community of Montes de María, Sucre, Colombia with more representation. It is representative the meat consumption like one of the sources of more importance to cure weakness and with aphrodisiac ends, of the three species of reptiles that are used Iguana iguana and Caiman crocodilus fuscus are of great value and its use includes besides the meat, the eggs and its fat. In general the detected uses were guided to the treatment of illnesses associated with inflammation, arthritis, weakness, fevers and sexual weakness.

\section{Palabras Clave:}

Etnozoología, fauna silvestre, Sucre,

Colombia.

\section{Resumen}

El presente estudio se evaluó el uso etnozoológicos dado a las especies de fauna silvestre que con mayor representación son consumidos por una comunidad rural de los montes de María, Sucre, Colombia. Se destaca el consumo de carne como una de las fuentes de mayor importancia para curar debilidad y con fines afrodisiacos, de las tres especies de reptiles que se usan Iguana iguana y Caiman crocodilus fuscus son de gran valor y su uso incluye además de la carne los huevos y su grasa. En general los usos detectados estaban orientados al tratamiento de enfermedades asociadas con inflamación, artritis, debilidad, fiebres y debilidad sexual. 


\section{Introducción}

En América Latina la fauna silvestre representa un importante papel como fuente de recursos alimenticios, medicinales, comerciales y deportivos que se apoyan en diversos factores de orden social, económico y cultural, con raíces comunes en el subdesarrollo y la marginalidad (MARES y OJEDA, 1984; REDFORD y ROBINSON, 1987; REDFORD y SANDERSON, 2000). Adicionalmente, se debe considerar que los residentes rurales, especialmente las comunidades aisladas dependen de abundantes recursos de caza para mantener un nivel nutricional adecuado (REDFORD y ROBINSON, 1987; DE LA OSSA y VOGT, 2010).

En cuanto a la importancia de la caza de subsistencia, por lo menos en 62 países del mundo contribuye aproximadamente con el $20 \%$ de la proteína animal en la dieta humana (ZAPATA, 2000). En ciertas partes selváticas, como el Amazonas, los indígenas satisfacen 100\% de su demanda proteica con la cacería (REDFORD y ROBINSON, 1987). Es necesario reconocer que en muchas regiones del mundo, la cacería local con fines de subsistencia es la única alternativa para la obtención de proteína de origen animal, ya que no es viable la utilización de animales domésticos (ALVARD, 2000; HILL y PADWE, 2000).

Los factores culturales desempeñan un papel fundamental en la conservación, en especial cuando se trata de uso que se le da a la biota silvestre. La conservación y el aprovechamiento son, en la práctica, inseparables (HERRERA, 1999). A escala regional, las tradiciones culturales de muchas comunidades campesinas e indígenas, muestran que el consumo y uso de la fauna silvestre es de vital importancia para su continuidad cultural (MANCERA y REYES, 2008). Por otro lado, la implementación de medidas restrictivas, ajenas a la realidad cultural, pueden ser ineficientes al tiempo que crean un escenario propicio que incentiva el uso no sustentable del recurso (ZWAHLEN, 1996).

Tradicionalmente el uso de los recursos naturales por parte de las comunidades indígenas y campesinas ha estado ligado al aprovechamiento selectivo de aquellas especies que tienen valor económico, tradicional y/o religioso (RACERO-CASARRUBIA et al., 2008). Muchos de estos grupos humanos tienen profundos conocimientos acerca del medio en que viven, así como de la diversidad de especies de plantas y animales (DERUYTTERE, 2007).

En resumen, COSTA-NETO et al. (2009) consideran que la Etnozoología es una rama de la ciencia enfocada en el estudio del conocimiento zoológico tradicional, de aquellos grupos humanos, indígenas, campesinos, pescadores artesanales, los cuales son llamados coloquialmente tradicionales, sin dejar de ser apreciado el conocimiento que poseen los habitantes de las grandes urbes respecto de los animales y sus usos.

El presente trabajo determina los patrones etnozoológicos de mayor ocurrencia en una comunidad campesina que habita la zona de amortiguación de los Montes de María, último refugio de bosque seco tropical que existe en el Caribe colombiano.

\section{Materiales y método}

Zona de trabajo: Ubicada en las estribaciones de los Montes de María, Sucre, Colombia, correspondiente al municipio de Colosó ( $9^{\circ} 30^{\prime} 24^{\prime \prime} \mathrm{N}$ y $75^{\circ} 21^{\prime} 48$ O) (Fig.1).

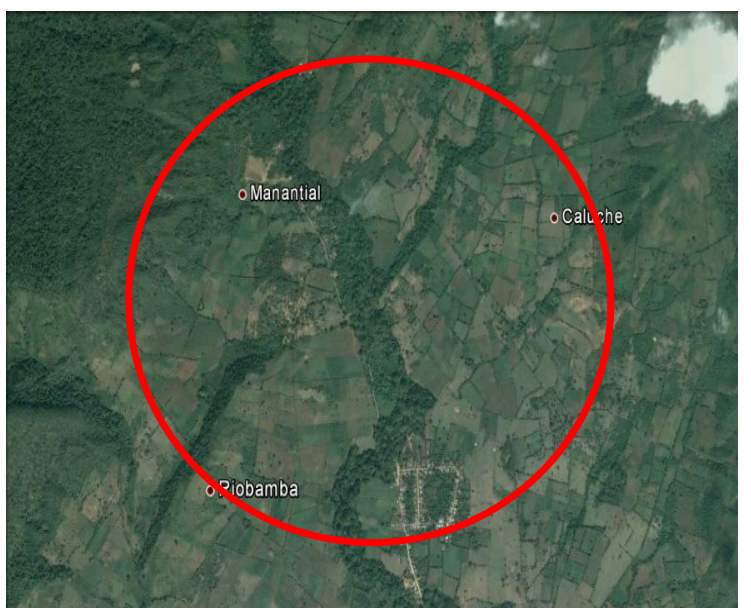

Figura 1. Zona de estudio (Versión libre Google earth)

Métodos: Se aplicaron un total de 45 encuestas semiestructuradas a pobladores rurales, mayores de 45 años y residentes tradicionales del lugar (MARGOLUIS y SALAFSKY, 1998). La muestra se obtuvo de una población previamente identificada de 80 moradores, usuarios directos del recurso, con margen de error del $10 \%$ y un nivel de confianza del $95 \%$. Las especies utilizadas, los volúmenes de caza así como los aspectos comerciales implicados fueron obtenidos de la encuestas.

Análisis de resultados: Los análisis realizados, dada las características de la información obtenida, son de tipo porcentual. La apreciación de la abundancia se fundamenta en una escala subjetiva de la detección que hacen en este caso los cazadores (RIDGELY y GWYNNE, 1989; STILES y BOHÓRQUEZ, 2000). 


\section{Resultados}

Nueve especies de vertebrados se referencian como usados etnozoológicamente por residentes en el área de estudio (Tabla 1) (Fig. 2). En la Tabla 2 se presenta la información relacionada con la cacería por especie e ingresos económicos obtenidos.

Tabla 1. Especies de vertebrados usados en el tratamiento de diferentes afecciones de salud humana

\begin{tabular}{|c|c|c|c|c|c|c|}
\hline Especie & Afección & Carne & Grasa & Huevos & Vísceras & Total \\
\hline \multirow{7}{*}{$\begin{array}{l}\text { Iguana iguana } \\
\text { (iguana) }\end{array}$} & Inflamación & 0 & 12 & 0 & 0 & 12 \\
\hline & Artritis & 0 & 10 & 0 & 0 & 10 \\
\hline & Debilidad & 20 & 0 & 45 & 0 & 65 \\
\hline & Fiebres & 10 & 0 & 0 & 0 & 10 \\
\hline & Afrodisiaco & 20 & 0 & 45 & 0 & 65 \\
\hline & Total & 50 & 22 & 90 & 0 & 162 \\
\hline & $\%$ & 30,9 & 13,6 & 55,5 & 0 & 100 \\
\hline \multirow{7}{*}{$\begin{array}{c}\text { Caiman crocodilus fuscus } \\
\text { (babilla) }\end{array}$} & Inflamación & 5 & 45 & 0 & 0 & 50 \\
\hline & Artritis & 45 & 45 & 0 & 0 & 90 \\
\hline & Debilidad & 15 & 0 & 28 & 0 & 43 \\
\hline & Fiebres & 0 & 2 & 0 & 0 & 2 \\
\hline & Afrodisiaco & 38 & 0 & 35 & 0 & 73 \\
\hline & Total & 103 & 92 & 63 & 0 & 258 \\
\hline & $\%$ & 39,9 & 35,7 & 24,4 & 0 & 100 \\
\hline \multirow{7}{*}{$\begin{array}{l}\text { Hydrochoerus hydrochaeris } \\
\text { (ponche) }\end{array}$} & Inflamación & 0 & 12 & 0 & 0 & 12 \\
\hline & Artritis & 0 & 22 & 0 & 0 & 22 \\
\hline & Debilidad & 40 & 0 & 0 & 3 & 43 \\
\hline & Fiebres & 1 & 0 & 0 & 0 & 1 \\
\hline & Afrodisiaco & 33 & 0 & 0 & 0 & 33 \\
\hline & Total & 74 & 34 & 0 & 3 & 111 \\
\hline & $\%$ & 66,7 & 30,6 & 0 & 2,7 & 100 \\
\hline \multirow{7}{*}{$\begin{array}{l}\text { Cuniculus paca } \\
\text { (guartinaja) }\end{array}$} & Inflamación & 0 & 0 & 0 & 0 & 0 \\
\hline & Artritis & 0 & 15 & 0 & 0 & 15 \\
\hline & Debilidad & 26 & 0 & 0 & 3 & 29 \\
\hline & Fiebres & 2 & 4 & 0 & 2 & 8 \\
\hline & Afrodisiaco & 26 & 1 & 0 & 4 & 31 \\
\hline & Total & 54 & 20 & 0 & 9 & 83 \\
\hline & $\%$ & 65,0 & 24,1 & 0 & 10,9 & 100 \\
\hline \multirow{7}{*}{$\begin{array}{l}\text { Dasyprocta punctata } \\
\text { (ñeque) }\end{array}$} & Inflamación & 0 & 4 & 0 & 0 & 4 \\
\hline & Artritis & 16 & 7 & 0 & 0 & 23 \\
\hline & Debilidad & 38 & 0 & 0 & 2 & 40 \\
\hline & Fiebres & 6 & 7 & 0 & 0 & 13 \\
\hline & Afrodisiaco & 28 & 0 & 0 & 3 & 31 \\
\hline & Total & 88 & 18 & 0 & 5 & 111 \\
\hline & $\%$ & 79,3 & 16,2 & 0 & 4,5 & 100 \\
\hline \multirow{7}{*}{$\begin{array}{l}\text { Tamandua mexicana } \\
\text { (oso hormiguero) }\end{array}$} & Inflamación & 0 & 15 & 0 & 0 & 15 \\
\hline & Artritis & 2 & 24 & 0 & 0 & 26 \\
\hline & Debilidad & 15 & 0 & 0 & 0 & 15 \\
\hline & Fiebres & 0 & 0 & 0 & 0 & 0 \\
\hline & Afrodisiaco & 0 & 9 & 0 & 0 & 9 \\
\hline & Total & 17 & 48 & 0 & 0 & 65 \\
\hline & $\%$ & 26,1 & 73,9 & 0 & 0 & 100 \\
\hline \multirow{7}{*}{$\begin{array}{l}\text { Choloepus hoffmanni } \\
\text { (perico ligero) }\end{array}$} & Inflamación & 0 & 19 & 0 & 0 & 19 \\
\hline & Artritis & 0 & 21 & 0 & 0 & 21 \\
\hline & Debilidad & 32 & 0 & 0 & 0 & 32 \\
\hline & Fiebres & 7 & 15 & 0 & 0 & 22 \\
\hline & Afrodisiaco & 28 & 0 & 0 & 0 & 28 \\
\hline & Total & 67 & 55 & 0 & 0 & 122 \\
\hline & $\%$ & 54,9 & 45,1 & 0 & 0 & 100 \\
\hline \multirow{7}{*}{$\begin{array}{l}\text { Boa constrictor } \\
\text { (boa) }\end{array}$} & Inflamación & 0 & 45 & 0 & 0 & 45 \\
\hline & Artritis & 0 & 22 & 0 & 0 & 22 \\
\hline & Debilidad & 0 & 0 & 0 & 0 & 0 \\
\hline & Fiebres & 0 & 0 & 0 & 0 & 0 \\
\hline & Afrodisiaco & 0 & 0 & 0 & 0 & 0 \\
\hline & Total & 0 & 67 & 0 & 0 & 67 \\
\hline & $\%$ & 0 & 100 & 0 & 0 & 100 \\
\hline \multirow{7}{*}{$\begin{array}{l}\text { Columba livia } \\
\text { (paloma) }\end{array}$} & Inflamación & 0 & 0 & 0 & 0 & 0 \\
\hline & Artritis & 0 & 0 & 0 & 0 & 0 \\
\hline & Debilidad & 45 & 0 & 3 & 0 & 48 \\
\hline & Fiebres & 45 & 0 & 2 & 0 & 47 \\
\hline & Afrodisiaco & 0 & 0 & 0 & 0 & 0 \\
\hline & Total & 90 & 0 & 0 & 5 & 95 \\
\hline & $\%$ & 94,7 & 0 & 0 & 5,3 & 100 \\
\hline
\end{tabular}

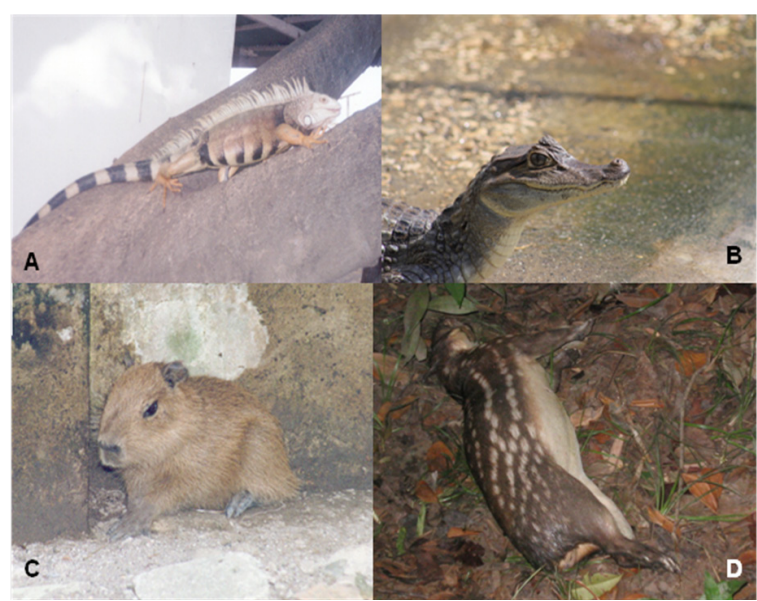

Figura 2. Algunas de las especies que se registran en este estudio. A (I. iguana), B (C. crocodilus fuscus), C ( $H$. hydrochaeris), $D$ (Cuniculus paca) (Fotos autores).

Tabla 2. Cacería de acuerdo con la época, cantidad de la captura, número de cazadores y precios (temporada seca $=\mathrm{S}, \mathrm{T}=$ todo el año).

\begin{tabular}{|c|c|c|c|c|c|c|}
\hline Especie & $E$ & $\mathbf{T}$ & стс & C & v\$ & I\$ \\
\hline $\begin{array}{l}\text { Iguana iguana } \\
\text { (iguana) }\end{array}$ & $S$ & 4 & 50 & 35 & 2 & 3.500 \\
\hline $\begin{array}{l}\text { Caiman crocodilus fuscus } \\
\text { (babilla) }\end{array}$ & $S$ & 4 & 2 & 9 & 10 & 180 \\
\hline $\begin{array}{l}\text { Hydrochoerus hydrochaeris } \\
\text { (ponche) }\end{array}$ & $S$ & 4 & 4 & 2 & 30 & 240 \\
\hline $\begin{array}{l}\text { Cuniculus paca } \\
\text { (guartinaja) }\end{array}$ & $\mathrm{T}$ & 12 & 6 & 12 & 25 & 1.800 \\
\hline $\begin{array}{l}\text { Dasyprocta punctata } \\
\text { (ñeque) }\end{array}$ & $\mathrm{T}$ & 12 & 8 & 12 & 15 & 1.440 \\
\hline $\begin{array}{l}\text { Tamandua mexicana } \\
\text { (oso hormiguero) }\end{array}$ & $S$ & 4 & 5 & 3 & 10 & 150 \\
\hline $\begin{array}{l}\text { Choloepus hoffmanni } \\
\text { (perico ligero) }\end{array}$ & $S$ & 4 & 10 & 5 & 8 & 400 \\
\hline $\begin{array}{l}\text { Boa constrictor } \\
\text { (boa) }\end{array}$ & $S$ & 4 & 6 & 2 & 20 & 240 \\
\hline $\begin{array}{l}\text { Columba livia } \\
\text { (paloma) }\end{array}$ & $\mathrm{T}$ & 12 & 30 & 6 & 2 & 360 \\
\hline \multicolumn{6}{|c|}{ Total ingreso } & 8.310 \\
\hline
\end{tabular}

$\mathrm{E}=$ Época; $\mathrm{T}=$ Temporada(meses);CTC= Capturas/temporada/cazador: C=Cazadores; V\$= venta (\$ US); I\$= Ingreso I/año (\$ US).

Los ingresos percibidos por actividad de caza se calculan US \$ 8.310 en total, lo que equivale a un ingreso anual por cazador, teniendo en cuenta que la muestra es de 45 encuestados, de US \$184,5. La percepción de ocurrencia de las especies utilizadas por esta comunidad se muestra en la Tabla 3. 
Tabla 3. Percepción de ocurrencia de las especies de fauna utilizadas con fines etnozoológicos.

\begin{tabular}{llc}
\hline \multicolumn{1}{c}{ Especie } & Rara Ocasional Abundante Muy abundante \\
\hline $\begin{array}{l}\text { lguana iguana } \\
\text { (iguana) }\end{array}$ & & $\mathrm{X}$ \\
$\begin{array}{l}\text { Caiman crocodilus fuscus } \\
\text { (babilla) }\end{array}$ & $\mathrm{X}$ & \\
$\begin{array}{l}\text { Hydrochoerus hydrochaeris } \\
\text { (ponche) }\end{array}$ & $\mathrm{X}$ & \\
$\begin{array}{l}\text { Cuniculus paca } \\
\text { (guartinaja) }\end{array}$ & $\mathrm{X}$ & \\
$\begin{array}{l}\text { Dasyprocta punctata } \\
\text { (ñeque) }\end{array}$ & $\mathrm{X}$ \\
$\begin{array}{l}\text { Tamandua mexicana } \\
\text { (oso hormiguero) }\end{array}$ & $\mathrm{X}$ \\
$\begin{array}{l}\text { Choloepus hoffmanni } \\
\text { (perico ligero) }\end{array}$ & $\mathrm{X}$ \\
$\begin{array}{l}\text { Boa constrictor } \\
\text { (boa) }\end{array}$ & $\mathrm{X}$ \\
$\begin{array}{l}\text { Columba livia } \\
\text { (paloma) }\end{array}$ & \\
\hline
\end{tabular}

\section{Discusión}

Los conocimientos tradicionales de los pueblos indígenas y de las comunidades locales van ganando atención global, debido a que complementan el conocimiento científico en diferentes áreas, dentro de las cuales se pueden destacar las evaluaciones de impacto ambiental, manejo de recursos y desarrollo sostenible (ZWAHLEN, 1996; SANTOS-FITA y COSTA-NETO, 2007). Las culturas tradicionales poseen modelos cognitivos de manejo de los recursos naturales que pueden mostrar valiosas vías de utilización alternativa del ambiente (SCHEPS, 1993) y entre ellas la fauna silvestre ocupa destacada posición.

Las tradiciones populares ejercen fuerte impacto sobre la conservación de la fauna, se tiene, por ejemplo, el caso del gavilán bebehumo (Buteo platypterus), los machos se capturan para venderlos (Col. \$5.000 o US \$2,5) en Chimá, Córdoba, con la intención de cruzarlo con gallinas de riña y obtener gallos de pelea más bravíos (SALAZAR-MEJíA, 2008)... ¿ ¿mito o realidad?... lo cierto que es que se practica.

También se cree que Iguana iguana puede aparearse con serpientes, razón que hace que su carne no sea apetecida (DE LA OSSA, 1995). Pero no se entiende el porqué del consumo de los huevos, si en esencia sería más representativo el tabú. Se cree que al abrir y extraer los huevos de I. iguana, rellenando la herida con hojarasca el animal sanará, lo cual es improbable, muestreos sistemáticos a través de 10 años que involucran la captura y liberación de más de 15.000 hembras ovadas no han mostrado una sola que presente cicatrices que lo demuestren (DE LA OSSA, 1995).
Llama la atención el uso medicinal dado a Bradypus variegatus y Choloepus hoffmanni en la región del Chocó, se identifica que algunas partes del cuerpo de las dos especies de perezosos, como el pelo y la grasa, se emplean para curar enfermedades como las hemorroides; además, ayuda a facilitar el embarazo; las uñas se utilizan como cicatrizante y la sangre caliente para curar la asfixia (PALACIOS-MOSQUERA et al., 2010).

A muchas especies de mamíferos silvestres se les aprecia por su valor en medicina tradicional. Por ejemplo, la sangre de Dasypus novemcinctus (armadillo) es utilizada para el tratamiento del asma; la vesícula biliar de Cuniculus paca (guartinaja) es usada por curanderos para el tratamiento de mordeduras de serpientes, y el caldo de carne de Choloepus hoffmanni (perezoso de dos dedos o perico ligero) se emplea para fortalecer personas con debilidad (CASTAÑO y CORRALES, 2010).

Igualmente se reporta que la carne de Rhinella marina (sapo común) se usa en aplicaciones dermatológicas y como cicatrizante; Bothropx asper (mapaná) y Lachesis muta (verrugoso) y Porthidium nasutum (patoco), su hiel se utiliza como antiofídico; Caiman crocodilus (babilla), su grasa como antiasmático; Cuniculus paca (guartinaja), la hiel como analgésico; Proechimys semispinosus (rata de monte) y Hoplomys gymnurus (rata espinosa), el hígado es tenido como estimulante hormonal del parto; las astas de Mazama americana (venado) como cicatrizante; marsupio e hígado de Didelphis marsupialis (zorra chucha) como estimulante hormonal del parto; sangre y cola de Dasypus novemcintus (armadillo) como antiasmático y contra la otitis; la grasa corporal de Tamandua mexicana (oso hormiguero) para la artritis; pene y testículos de Potos flavus (cusumbo, kinkayú) como afrodisiacos (CUESTASRíO et al., 2007).

En este estudio se destaca el papel afrodisíaco que se le concede a la carne y huevos de I. iguana y de $C$. crocodilus fuscus; igualmente a la carne de $H$. hydrochaeris, $C$. paca, D. punctata, T. mexicana y C. hoffmanni. Lo cual concuerda en términos generales con lo planteado por estudios similares para América (PÉREZ-GIL et al., 1996; OTCA, 1999).

En cuanto a la percepción de la abundancia de las especies objeto de estudio, se evidencia que exceptuando a Columbia livia domestica, cuyo carácter de invasora es reconocido (VILLALBA-SÁNCHEZ y DE LA OSSALACAYO, 2014), las demás especies no son abundantes, lo que podría inferir un deterioro de sus poblaciones que debería ser estudiado de una manera más profunda y puntual. Quedaría por comprobar medicamente, si el uso dado a la fauna silvestre que se determina en este estudio ofrece buenos resultados clínicos y más allá se debería investigar sobre las sustancias que actúan en tal sentido. 


\section{Referencias}

ALVARD, M. 2000. The impact of traditional subsistence hunting and trapping populations: data from Wana horticulturalists of Upland Central Sulawesi, Indonesia. En: Robinson JG, Redford KH. (Eds.). Neotropical Wildlife Use and Conservation. Chicago University Press. Chicago, USA: 214-230.

CASTAÑO, J.H.; CORRALES, J.D. 2010. Mamíferos de la cuenca del río la miel (Caldas): diversidad y uso cultural. Bol.Cient.Mus.Hist.Nat. 2010; 14(1):56-75.

COSTA, E.M.; SANTOS, F.D.; VARGAS, C.M. 2009. Manual de Etnozoología, una guía teórico-práctica para investigar la interconexión del ser humano con los animales. Editorial Tundra. España. .

CUESTAS-RÍO, E.Y.; VALENCIA-MAZO, J.D.; JIMÉNEZ-ORTEGA, AM. 2007. Aprovechamiento de los vertebrados terrestres por una comunidad humana en bosque tropicales (Tutumendo, Chocó, Colombia). Revista Institucional Universidad Tecnológica del Chocó: Investigación, Biodiversidad y Desarrollo 26 (2):37-43.

DE LA OSSA, V.J. 1995. Estudio Ecológico sobre Reproducción de Iguana iguana (Linnaeus, 1758) y su Utilización como Recurso Alimenticio en San Marcos (Sucre), Colombia. Bogotá. Tesis Especialista en Ecología, Medio Ambiente y Desarrollo. Universidad INCCA de Colombia. Facultad de Posgrados; 1995

DE LA OSSA, V.J.; VOGT, R.C. 2010. Efecto de sustitución: una expresión del agotamiento poblacional de quelonios en Barcelos, Amazonas, Brasil. Rev. Asoc. Col. Cienc. 22:61-67.

DERUYTTERE, A. 2007. Pueblos indígenas y desarrollo sostenible: el papel del Banco Interamericano de Desarrollo. Unidad de Pueblos Indígenas y Desarrollo Comunitario, Departamento de Desarrollo Sostenible. Washington D.C; 1997.

HERRERA, E. 1999. Comportamiento, conservación y manejo de fauna silvestre: el caso del capibara en Venezuela. Etología 7:41-46.

HILL, K.; PADWE, J. 2000. Sustainability of Aché Hunting in the Mbaracayú Reserve, Paraguay. En: Robinson JG, Bennett EL. (Eds.). Hunting for Sustainability in Tropical Forests. Columbia University. New York, USA: 79-105.

MANCERA, N.; REYES, O. 2008. Comercio de fauna silvestre en Colombia. Rev. Fac. Nal. Agr. Medellín 2008; 61(2):4618-4745.

MARES, M.A.; OJEDA, R.A. 1984. Faunal commercialization as a factor in South American rarefaction. BioScience 34:580-584.

MARGOLUIS, R.; SALAFSKY, N. 1998. Measures of success: designing, managing, a $\quad$ d m o n it o ring conservation and development projects. Island Press. Washington, DC.

ORGANIZACIÓN DEL TRATADO DE COOPERACIÓN AMAZÓNICA (OTCA). 1999. Conservación y uso de la fauna silvestre en áreas protegidas de la Amazonía. Caracas, Venezuela.

PALACIOS-MOSQUERA, L.; MENA-ROJAS, O.P.; SÁNCHEZ-LOZANO, L.E. 2010. Uso tradicional de osos perezosos (Bradypus variegatus y Choloepus hoffmanni) en seis municipios del departamento del Chocó, Colombia. Bioetnia $7(1): 4-9$

PÉREZ-GIL, S.R.; JARAMILLO, M.F.; MUÑIZ, S.A.M.; TORRES, G.M.GT. 1996. Importancia económica de los vertebrados de México. CONABIO. México, D.F.

RACERO-CASARRUBIA, J.S.; VIDAL, C.C.; RUIZ, O.D.; BALLESTEROS, C.J. 2008. Percepción y patrones de uso de la fauna silvestre por las comunidades indígenas Embera-Katíos en la cuenca del río San Jorge, zona amortiguadora del PNN-Paramillo. Revista de Estudios Sociales 31:118-131. 
REDFORD, K.H.; ROBINSON, J.G. 1987. The game of choice: patterns of Indian and colonist hunting in the neotropics. American Anthropologist 89:650-667.

REDFORD, K.H.; SANDERSON, S. 2000. Stracting human from nature. Conservation Biology 14 (5):1362-1364.

RIDGELY, R.S.; GWYNNE, J.A. 1989. A guide to the birds of Panama. Princeton University Press. Princeton, NJ, USA.

SALAZAR-MEJÍA, I. 2008. La economía de la Ciénaga Grande del Bajo Sinú: lugar encantado de las aguas. Documentos de Trabajo Sobre Economía Regional 102. Banco de la República. Centro de Estudios Económicos Regionales CEER. Cartagena, Colombia; 2008.

SANTOS-FITA, D.; COSTA-NETO, E.M. 2007. As interações entre os seres humanos e os animais: a contribuição da etnozoologia. Biotemas 2007; 20(4):99-110.

SCHEPS, R. 1993. La science sauvage. Des savoirs populaires aux ethnosciences. Éditions du Seuil. Paris, France; 1993.

STIIES, F.G.; BOHÓRQUEZ, C.I. 2000. Evaluando el estado de la biodiversidad: el caso de la avifauna de la serranía de las Quinchas, Boyacá, Colombia. Caldasia 22 (1):61-92.

VILLALBA-SÁNCHEZ, C.; DE LA OSSA-LACAYO, A. 2014. Columba livia domestica Gmelin, 1789: plaga o símbolo. Rev. Colombiana cienc. Anim. (Colombia). 6 (2):424-433.

ZAPATA, R.G. 2000. Sustentabilidad de la cacería de subsistencia: El caso de cuatro comunidades quichuas en la amazonía nororiental ecuatoriana. Mastozoología Neotropical. J. Neotrop. Mammal 8 (1):59- 66.

ZWAHLEN, R. 1996. Traditional methods: a guarantee for sustainability? Indigenous Knowledge and Development Monitor 1996; 4(3): 1-7. 